\title{
ASSESSMENT OF FETAL LEFT CARDIAC ISOVOLUMIC RELAXATION TIME IN APPROPRIATE AND SMALL-FOR- GESTATIONAL-AGE FETUSES
}

\author{
P. TSYVIAN, ${ }^{\ddagger}{ }^{\ddagger}$ K. MALKIN ${ }^{\dagger}$ and J. W. WLADIMIROFF ${ }^{\ddagger}$ \\ ${ }^{\dagger}$ I aboratory of Biophysics, Mother and Child Institute, Yekaterinburg, Russia; and \\ Department of Obstetrics and Gynecology, Erasmus University, Rotterdam, The Netherlands
}

(Received 20 July 1994; in final form 2 December 1994)

\begin{abstract}
Left ventricular isovolumic relaxation time was studied in 22 appropriate-for-gestational-age fetuses (AGA, 26-40 wk) and 12 small-for-gestational-age fetuses (SGA, 29-37 wk). Left ventricular isovolumic relaxation time was determined from the interval between aortic valve closure and maximal left atrial dimension by $M$-mode, and from the interval between aortic valve closure artefact and onset of transmitral flow by pulsed Doppler. Mean left ventricular isovolumic relaxation time by M-mode (36 \pm 6 $\mathrm{ms})$ and by pulsed Doppler $(49 \pm 10 \mathrm{~ms})$ were significantly different $(p<0.05)$ in AGA while this was not so in SGA $(56 \pm 10 \mathrm{~ms}$ vs. $60 \pm 8 \mathrm{~ms})$. A significant difference $(p<0.05)$ in mean left ventricular isovolumic relaxation time by M-mode existed between AGA (36 \pm 6 ms) and SGA ( $56 \pm 10$ ms), whereas this was not so for pulsed Doppler ( $48 \pm 10 \mathrm{~ms}$ vs. $60 \pm 8 \mathrm{~ms}$ ). Mean left ventricular isovolumic relaxation time by Doppler was significantly larger (mean difference $14 \pm 8 \mathrm{~ms} ; p<0.05$ ) than by M-mode in AGA. However, there was no difference in mean left ventricular isovolumic relaxation time between the two ultrasound modalities in SGA. These data suggest synchronization of mitral cusp separation and transmitral blood flow in the SGA fetus. We speculate that, in the SGA fetus, delayed left ventricular isovolumic relaxation time may reflect cardiac diastolic dysfunction.
\end{abstract}

Key Words: Fetal cardiac isovolumic relaxation, M-mode, Doppler ultrasound, Small-for-gestational-age fetus, Pregnancy-induced hypertension.

\section{INTRODUCTION}

Doppler studies of fetal cardiac and peripheral arterial flow velocity waveforms have been reported to identify small-for-gestational-age (SGA) fetuses, which are at risk for chronic hypoxemia (Groenenberg et al. 1989; Trudinger et al. 1987). Reduced end-diastolic flow velocities in the fetal aorta and umbilical artery have been associated with increased peripheral vascular resistance, suggesting decreased placental perfusion (Rizzo et al. 1992). Reduced peak systolic flow velocities have been demonstrated at the cardiac level, which may be the result of reduced volume flow (decreased preload), increased afterload or impaired contractility of the fetal myocardium (Al-Ghazali et al. 1988; Groenenberg et al. 1989).

The fetal heart mainly operates according to the Frank-Starling mechanism and the leading determi-

Address correspondence to: Prof. J. W. Wladimiroff, M.D., Ph.D., Department of Obstetrics and Gynecology. Academic Hospital Rotterdam-Dijkzigt, Erasmus University, Dr. Molewaterplein 40, 3015 GD, Rotterdam, The Netherlands. nant of fetal cardiac performance is the filling of the ventricles (Romero et al. 1972; Rudolph 1985). Marked enhancement of the late diastolic (atrial) phase of ventricular filling has been observed in the smallfor-gestational-age fetus, which was considered indirect evidence of increased myocardial stiffness (Rizzo et al. 1988).

Ventricular diastole consists of an isovolumic relaxation phase and a ventricular filling phase. During isovolumic relaxation a rapid drop in left ventricular pressure takes place. Because there are no changes in ventricular volume at this time, the major determinants of chamber pressure change are force decay in the contractile units, elastic recoil of the ventricles and afterload (Brutsaert and Sys 1989; Nikolic et al. 1988). Whereas there is some information available on ventricular filling (Rizzo et al. 1988), no data exist on isovolumic relaxation time during fetal life. Left ventricular isovolumic relaxation time can be assessed by two different ultrasound techniques: M-mode and pulsed Doppler ultrasound. They measure different aspects of atrioventricular activity. 
The objective of the present study was to investigate left ventricular isovolumic relaxation time in both appropriate and small-for-gestational-age fetuses. The reason for studying left ventricular isovolumic relaxation time was twofold: (i) left ventricular cardiac contractility and output are essential for adequate oxygen supply of both the fetal myocardium and the fetal brain; and (ii) simultaneous recording of pulmonary artery valve movement and right ventricular dimension, necessary for determining right isovolumic relaxation time, is as yet not feasible.

\section{MATERIALS AND METHODS}

Between 1 July 1993 and 1 February 1994, a total of 34 women from the outpatient department of the Mother and Child Institute, Yekaterinburg, consented to participate in the study. In each case, there was a singleton pregnancy, and pregnancy duration was determined from the last menstrual period and confirmed by ultrasonic measurement of the biparietal diameter between 14 and 18 weeks of gestation. Pregnancy was uneventful in 22 of 34 women. Pregnancy duration varied between 26 and 40 (median 36) weeks. Fetal upper-abdominal circumference was always situated between 10th and 90th percentile (Yudkin et al. 1987). There were no structural abnormalities. Small for gestational age, as expressed by an upper-abdominal circumference below the 5th percentile (Yudkin et al. 1987), was diagnosed in the remaining 12 pregnancies. Pregnancy duration ranged between 29 and 37 (median 34) weeks of gestation. Pregnancy-induced hypertension ( $\mathrm{PIH}$ ), defined as a diastolic blood pressure of $90 \mathrm{mmHg}$ or more, was present in 8 of 12 women; and pre-eclamptic toxemia, defined as PIH and proteinuria of $300 \mathrm{mg} / \mathrm{L}$ or more, existed in the remaining 4 women. There were no structural anomalies. Maternal age in both groups ranged between 18 and 37 years (median 27 years). All women were nonsmokers, and no medication was taken apart from iron tablets.

There are two established methods for determining the period of time for isovolumic relaxation of the left ventricle: the time between the end of left ventricular contraction and the beginning of left atrial filling. The time difference between aortic valve closure and maximal left atrial dimension can be assessed using M-mode. Doppler ultrasound can record simultaneously the aortic valve closure and mitral valve opening.

Left isovolumic relaxation time ( $\mathrm{ms}$ ) was determined using a combined curved-linear-array two-dimensional real-time, pulsed Doppler and $\mathrm{M}$-mode recording system (Aloka SSD 650, Aloka Industry Corp., Tokyo). The Doppler carrier frequency was 3.5 $\mathrm{MHz}$. The four-chamber view of the heart was visual- ized on a transverse cross-section. The Doppler sample volume was then placed immediately distal to the mitral valve leaflets. The high-pass filter was set at 100 $\mathrm{Hz}$. Doppler tracings were accepted when the angle between the Doppler cursor and the assumed flow direction was $10^{\circ}$ or less. On the Doppler waveform traces the left isovolumic relaxation time was determined from the artefact of aortic valve closure to the onset of transmitral flow (Fig. 1). The latter was taken as the point where the signal rose above the filter. Fetal heart rate (bpm) was calculated from the time difference between the onset of transmitral flow in two consecutive cardiac cycles.

Simultaneous $\mathbf{M}$-mode registration of aortic valve and left atrial wall movement allows determination of left isovolumic relaxation time by establishing the time interval between aortic valve closure and the onset of maximal left atrial dimension (Fig. 2). Fetal heart rate was calculated from the time difference between aortic valve closure in two consecutive cardiac cycles. Umbilical artery Doppler flow velocity waveforms were recorded from a free-floating loop of the umbilical cord for calculation of the systolic/diastolic (S/D) ratio as a measure of downstream impedance.

Analysis of Doppler waveforms and M-mode recordings was performed from hard copies using a personal computer Apricot XEN-S linked to a graphic tablet. Maximal left atrial dimension was determined by manually tracing the endocardial borderlines of the left atrium and subsequently storing each contour in the computer memory for digital measurements of maximum dimension. For each subject the mean of three consecutive cardiac cycles of acceptable technical quality was taken for calculation of the left isovolumic relaxation time according to the Doppler and M-mode modalities. The obstetrician responsible for clinical management was unaware of the data analysis.

All data are presented as mean \pm SD. Differences in left isovolumic relaxation time between AGA and SGA fetuses and between Doppler and M-mode recordings were determined using the paired Student's $t$ test. Linear correlation coefficients between isovolumic relaxation time as calculated from Doppler and $M$-mode recordings and heart rate gestational age (wk) and umbilical systolic/diastolic (S/D) ratio were determined. The level of statistical significance was chosen as $p<0.05$.

\section{RESULTS}

Mean fetal heart rate (FHR) was not significantly different between: (i) AGA and SGA fetuses (144 \pm $11 \mathrm{bpm}$ vs. $141 \pm 10 \mathrm{bpm}$ for M-mode, and $143 \pm 9$ bpm vs. $142 \pm 11 \mathrm{bpm}$ for Doppler); and (ii) the two ultrasound modalities in AGA and SGA fetuses (144 


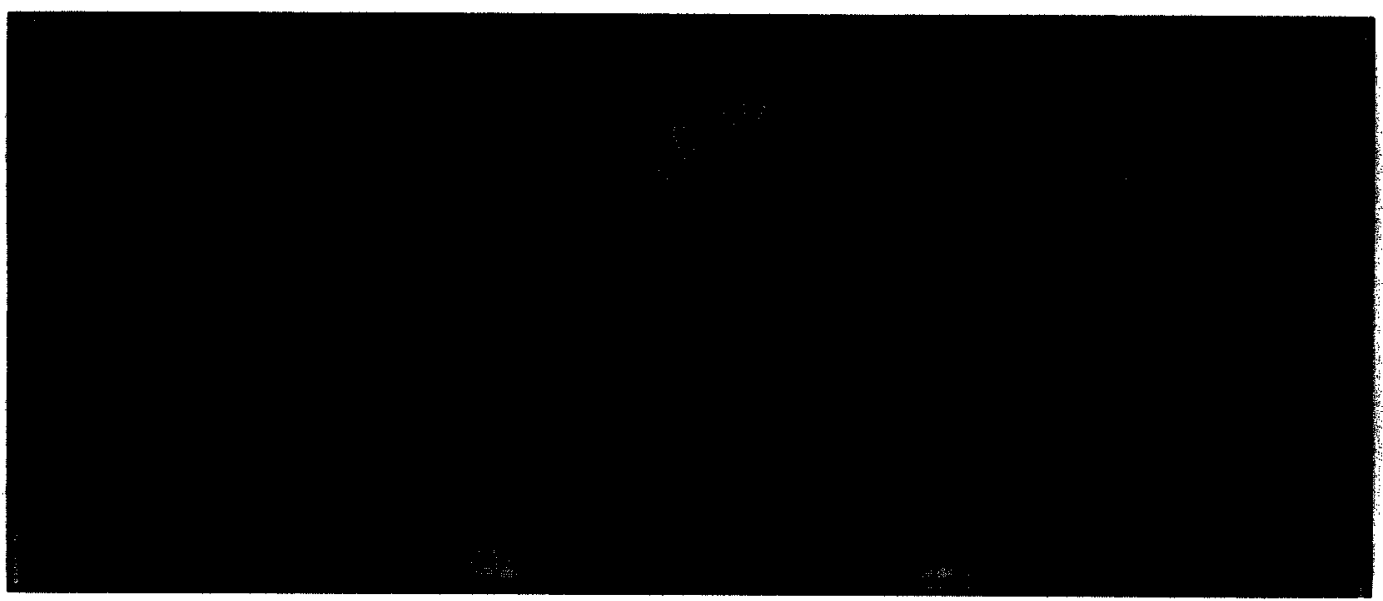

Fig. 1. Pulsed Doppler echocardiogram from a normally developing fetus at 32 weeks of gestation. Left isovolumic relaxation time is determined from the interval between aortic valve closure artefact (dotted line, see arrow) and the onset of transmitral flow (continuous line). " $E$ " represents the early component and " $A$ " represents the late component of the transmitral flow velocity waveform.

$\pm 11 \mathrm{bpm}$ vs. $143 \pm 9 \mathrm{bpm}$ in M-mode, and $141 \pm$ 10 bpm vs. $142 \pm 11$ bpm in Doppler mode). Doppler systolic/diastolic ratios calculated from the umbilical artery flow velocity waveforms of SGA fetuses were always situated above the 95th percentile (Ducey et al. 1987).

In the AGA fetus, mean left isovolumic relaxation time as measured from the M-mode recording (36 \pm $6 \mathrm{~ms})$ was significantly shorter $(p=0.04)$ than that measured by Doppler ultrasound ( $49 \pm 10 \mathrm{~ms})$. In the small-for-gestational-age fetus this difference was not significant ( $56 \pm 10 \mathrm{~ms}$ for M-mode vs. $60 \pm 8 \mathrm{~ms}$ for Doppler).

According to the M-mode method mean left isovolumic relaxation time ( $56 \pm 10 \mathrm{~ms})$ in the SGA fetus was significantly longer $(p=0.03)$ than in normally developing fetuses ( $36 \pm 6 \mathrm{~ms}$ ). According to Doppler ultrasound, the difference between SGA and AGA fetuses $(60 \pm 8 \mathrm{~ms}$ vs. $49 \pm 10 \mathrm{~ms})$ was not significant. In normal fetuses no correlation could be

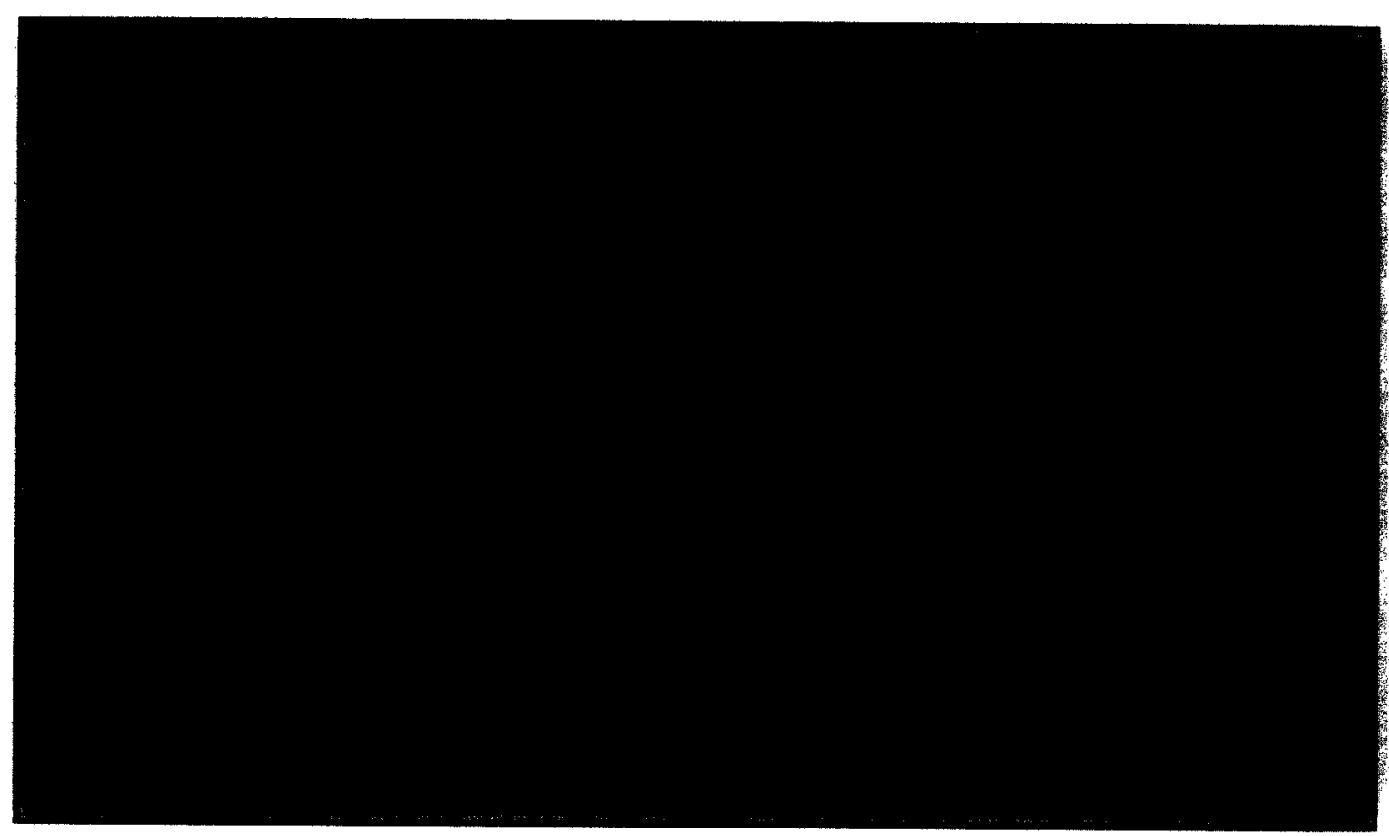

Fig. 2. M-mode echocardiogram from a normally developing fetus at 32 weeks of gestation. Left isovolumic relaxation time is determined from the interval between aortic valve closure (dotted line) and the onset of maximal left atrial dimension (continuous line). Ao = ascending aorta, $\mathrm{LA}=$ left atrium. 
Table 1. Coefficients of correlation $(r)$ between left ventricular isovolumic relaxation time (IRT) as measured by $\mathrm{M}$-mode (M) and pulsed Doppler (D) and gestational age, fetal heart rate and umbilical artery

systolic/diastolic ratio in appropriate (AGA) and small-for-gestational-age (SGA) fetuses.

\begin{tabular}{lccc}
\hline & $\begin{array}{c}\text { Gestational } \\
\text { age }\end{array}$ & $\begin{array}{c}\text { Fetal heart } \\
\text { rate }\end{array}$ & $\begin{array}{c}\text { Umbilical artery } \\
\text { systolic/diastolic } \\
\text { ratio }\end{array}$ \\
\hline IRT-M AGA & -0.26 & -0.14 & 0.20 \\
IRT-M SGA & 0.37 & $-0.49^{+}$ & -0.18 \\
IRT-D AGA & -0.14 & -0.25 & 0.04 \\
IRT-D SGA & $0.53^{*}$ & -0.29 & $-0.45^{+}$
\end{tabular}

$p<0.05$

demonstrated between left isovolumic relaxation time as observed by Doppler and $\mathbf{M}$-mode method and gestational age, FHR and umbilical artery S/D ratio (Table 1 ).

In the SGA fetuses a positive correlation ( $r=$ $+0.53, p=0.01$ ) was established between left isovolumic relaxation time (Doppler) and gestational age, and a negative correlation was determined between: (i) left isovolumic relaxation time (Mmodc) and FHR ( $r=0.49, p=0.03)$; (ii) left isovolumic relaxation time (Doppler) and umbilical S/D ratio $(r=-0.45, p=0.04)$ (Table 1). Data from SGA fetuses were too small to differentiate between pregnancy-induced hypertension $(n=8)$ and pre-eclamptic toxemia $(n=4)$.

\section{DISCUSSION}

In the normally developing fetus, left isovolumic relaxation time appears to be a stable parameter which has no relationship with fetal heart rate, gestational age and umbilical S/D ratio. However, a significant difference in left isovolumic relaxation time could be demonstrated between the M-mode and Doppler modality.

Data obtained from dog experiments have shown that the mitral valve reaches its maximal excursion (Epoint on M-mode scan) to start its mid-diastolic closing motion approximately $25 \mathrm{~ms}$ before the onset of transmitral peak flow (Yellin et al. 1981). These data on the onset of mitral cusp separation before mitral flow have led to the conclusion that the mitral valve is always under chordal constraint (Yellin et al. 1990).

Furthermore, it was shown that the configuration of the mitral valve apparatus during early diastole serves to increase local blood velocity and to decrease the pressure within the leaflets (Yellin et al. 1981). Therefore, as a result of mitral valve configuration the appropriate shape of the left atrium and decrease of atrial diastolic dimension may take place without ap- parent emptying of the atrium. From this follows that the recording of left isovolumic relaxation time by $\mathrm{M}$ mode and Doppler ultrasound represents two physiologically different processes: the measurement of the time between aortic valve closure and maximal left atrial dimension mainly reflects force decay in the myocardium and changes in force control of the mitral chordal constraint; the measurement of time between aortic valve closure and mitral valve opening mainly reflects temporal changes in transmitral pressure gradient. We suggest that the configuration of the mitral valve and concomitant leaflet separation may precede the onset of transmitral flow only in case of a normal (uniform) sequence of cardiac force decay and a normal transmitral pressure gradient.

In the SGA fetus an increase in left isovolumic relaxation time is demonstrated for M-mode, which mainly represents force decay in the myocardium.

It is known that, in mammalian myocardium, there are three interacting determinants of relaxation: (i) afterload; (ii) inactivation of contraction (decay of active force); and (iii) nonuniform distribution of afterload and inactivation in space and time (Brutsaert and Sys 1989). Intrauterine growth retardation has been associated with a raised afterload (Rizzo et al 1992; Trudinger et al. 1987) as was also shown by raised umbilical artery S/D ratios in the present study. Chronic hypoxemia, which may occur in the SGA fetus can cause significant impairment of cardiac relaxation as a result of delayed inactivation of myocardial contraction and impairment of intracellular $\mathrm{Ca}^{++}$transporting systems (Silverman et al. 1991). Chronic intrauterine hypoxemia may also result in increased diastolic left ventricular wall thickness (Vasenina 1990), which in turn may induce nonuniform distribution of afterload and inactivation of cardiac contraction. In the SGA fetus, all three above-mentioned factors may therefore be held responsible for the observed lengthening of left isovolumic relaxation time as obtained from the M-mode scan.

No significant difference was established in the SGA fetus regarding left isovolumic relaxation time when comparing M-mode and Doppler ultrasound, suggesting synchronization of mitral cusp separation and transmitral blood flow. Intrauterine growth retardation secondary to uteroplacental insufficiency is characterised by selective changes in peripheral vascular resistance resulting in a shift in cardiac output in favour of the left ventricle (Rizzo et al. 1992). In the presence of raised left ventricular afterload due to peripheral vasoconstriction, increase in left ventricular output may only be possible as a result of a substantial rise in left ventricular cardiac filling and transmitral atrioventricular pressure gradient. This would lead to a shorter time interval between mitral cusp separation 
and onset of transmitral blood flow. For example, more temporal synchronisation between these events. A similar observation has been made in adults in the presence of a high left atrioventricular pressure gradient as a result of mitral stenosis (Lee et al. 1990). An alternative explanation to the synchronization of mitral cusp separation and transmitral blood flow in the SGA fetus could be a raised intra-atrial pressure required to overcome the intraventricular pressure and open the mitral valve in response to fetal hypertension.

The influence of the transmitral pressure gradient upon transmitral blood flow can also explain the absence of a significant difference in left isovolumic relaxation time determined by Doppler ultrasound between normally developing and SGA fetuses. As was mentioned earlier, the recording of left isovolumic relaxation time by $\mathbf{M}$-mode and Doppler ultrasound represents two physiologically different processes; whereas the $\mathbf{M}$-mode representation is able to determine the delay in force decay in the SGA fetus, the Doppler modality fails to do so. The Doppler representation mainly reflects the increase in transmitral pressure gradient which leads to an early onset of transmitral flow and therefore masks the effect of force decay on transmitral blood flow. A practical implication of these observations could be that monitoring cardiac performance in SGA infants associated with maternal pregnancy-induced hypertension should be performed using the M-mode technique as this is a more sensitive indicator of cardiac diastolic dysfunction.

The negative correlation between left isovolumic relaxation time ( $\mathrm{M}$-mode) and heart rate in the SGA fetus may be explained by the greater temporal synchronisation of mitral cusp separation and transmitral blood flow. It could also mean that, in the SGA fetus, there is a larger contribution by the left atrioventricular pressure gradient to the opening of the mitral valve than in normal fetal development. The positive correlation which was observed between left isovolumic relaxation time (Doppler) and gestational age in the SGA fetus may be the result of prolonged diastolic failure as expressed by sustained cardiac force decay and/or increase in myocardial stiffness due to chronic hypoxemia.

\section{CONCLUSION}

Left ventricular isovolumic relaxation time is increased in the SGA fetus. The time period between aortic closure and maximal left atrial dimension is sig- nificantly increased, but there is only a marginal effect on timing of the mitral valve opening. The increase in left isovolumic relaxation time as determined by $\mathbf{M}$ mode ultrasound suggests cardiac diastolic dysfunction. We speculate that the similarity of left isovolumic relaxation time, as determined by both recording modalities, may reflect a possible rise in transmitral pressure gradient in the small-for-gestational-age fetus as a result of enhanced left ventricular venous return.

Acknowledgements - P. Tsyvian, M.D., Ph.D., is recipient of a research fellowship from the Dutch Research Council (NWO), Grant 07-13-091.

\section{REFERENCES}

Al-Ghazali, W.; Chapman, M. G.; Allan, L. D. Doppler assessment of the cardiac and uteroplacental circulation in normal and complicated pregnancies. Br. J. Obstet. Gynaecol. 96:575-580; 1988.

Brutsaert, D. L.; Sys. S. U. Relaxation and diastole of the heart. Physiol. Rev. 69:1228-1315; 1989.

Ducey, J.; Schulman, H.; Farmakides, G.; Rochelson, B.; Bracero, L., et al. A classification of hypertension in pregnancy based on Doppler velocimetry. Am. J. Obstet. Gynecol. 156:1223-1226: 1987.

Groenenberg, I. A. L.; Wladimiroff, J. W.: Hop. W. C. J. Fetal cardiac and peripheral arterial flow velocity waveforms in intrauterine growth retardation. Circulation 80:1711-1717; 1989.

Lee, C. H.; Vancheri, F.; Josen, M. S.; Gibson, D. G. Discrepancies in the measurement of isovolumic relaxation time: a study comparing M-mode and Doppler echocardiography. Br. Heart $\mathbf{J}$. 64:214-218; 1990.

Nikolic, S.; Yellin, E. L.; Tamura, K. Passive properties of canine left ventricle: Diastolic stiffness and restoring forces. Circ. Res. 62:1210-1222; 1988.

Rizzo, G.; Arduini, D.; Romanini, C.; Mancuso. S. Doppler echocardiographic assessment of atrioventricular velocity waveforms in normal and small for gestational age fetuses. Br. J. Obstet. Gynaecol. 95:65-70; 1988

Rizzo, G.; Arduini, D.; Romanini, C. Doppler aechocardiographic assessment of fetal cardiac function. Ultrasound Obstet. Gynecol. $2: 434-445 ; 1992$.

Romero, T.; Covell, J.; Friedman, W. F. A comparison of pressurevolume relation of the fetal, newborn, and adult heart. Am. J. Physiol 222:H1285-H1290; 1972.

Rudolph. A. M. Distribution and regulation of blood flow in the fetal and neonatal lamb. Circ. Res. 57:811-821; 1985.

Silverman, H. S.; Ninomiya, M.; Blank, P. S.; Hano, O.; Miyata, $\mathrm{H}$., et al. A cellular mechanism for impaired posthypoxic relaxation in isolated cardiac myocytes. Circ. Res. 69:196 208; 1991.

Trudinger, B. J.; Stevens, D.; Conelly. A.; Hales, R. J.; Alexander. $\mathrm{J}$., et al. Umbilical artery flow velocity waveforms and placental resistance: The effect of embolization of the umbilical circulation. Am. J. Obstet. Gynecol. 157:1443-1448; 1987.

Vasenina, A. D. Systolic and diastolic function in the heart of newborn infants in normal conditions and after perinatal asphyxia. Ph.D. dissertation; Medical Institute, Sverdlovsk, Russia: 1990.

Yellin, E. L.; Peskin, C.: Yoran, C. Mechanism of mitral valve motion during diastole. Am. J. Physiol. 241:H389-H400; 1981.

Yellin, E. L.; Nikolic, S.; Frater, R. W. M. Left ventricular filling dynamics and diastolic function. Progr. Cardiovasc. Dis. 32:247$271 ; 1990$.

Yudkin, P. L.; Aboulfa, M.; Eyre, J. A.; Redman, C. W. G.; Wilkinson, A. R., et al. New birthweight and head circumference centiles for gestational ages 24 to 42 weeks. Early Hum. Devel. $15: 45-52 ; 1987$ 